\title{
Clinical and laboratory parameters in a cohort of CLL patient: a single centre experience
}

\begin{abstract}
Introduction: For patients diagnosed with CLL, it is necessary to clinically stage the disease by both the Rai and Binet systems. Although both staging systems are related to systemic spread, disease severity, and prognosis, concurrent genomic and molecular aberrations could alter the clinical course and outcome and could influence treatment strategy and clinical trial selection.
\end{abstract}

Objective: The objective of this study was to determine the association of various clinical and laboratory parameters in CLL patients.

Method: All the patients diagnosed as Chronic Lymphocytic Leukemia from January 2010 to July 2013 were included. Institutional review board approved the study. Informed consent was obtained. Clinical and laboratory findings were retrieved retrospectively from patients file. SPSS ver 17 was used for statistical analysis.

Results: A total of 49 patents were enrolled including 35(71\%) males and $14(28.5 \%)$ females. Mean age was 60 years. Out of 49, 36 patients were symptomatic including fever (19), weight loss (15), and sweating (02). Visceromegaly was seen in 19 patients $(38.7 \%$ ) (Hepatomegaly in 07 and splenomegaly in 12 patients) and 16 patient had lymphadenopathy. According to Rai staging, stage 0 was seen $25(51 \%)$ patients, stage I in $15(30.6 \%)$, stage II in $11(22.4 \%)$, stage III in $14(28.5 \%)$ and stage IV in $12(24.4 \%)$ patients. According to Binet staging $04(8.1 \%)$ in stage A, 04(8.1\%) in stage B and $17(34.6 \%)$ noted. On bone marrow biopsy pattern of infiltration was Interstitial in (14), diffuse in (21), nodular in (07), interstitial to diffuse in (07) seen. Immunohistochemistry panel showed anti CD5, CD20, CD23, CD79a in almost all the cases. ZAP 70 positivity was seen in $17(34.6 \%)$ patients.

Conclusion: In our center most of the patients were in Rai/Binet 0/A stage. About 1/3 showed ZAP 70 positivity which indicates poor prognosis. All the CLL patient should not only be staged but also other genomic markers be applied to stratify the management accordingly.

Keywords: rai/binet staging; ZAP 70, visceromegaly, bone marrow biopsy, ECOG
Volume 5 Issue 4 - 2017

\section{Noorul Ain Fareed, Mehwesh Taj, Bushra Kaleem, Nadeem Muhammad, Rizwan Nabi Qureshi, Tahir Shamsi}

Department of clinical Hematology and Bone Marrow Transplantation, National Institute of Blood Disease \& Bone Marrow Transplantation, Pakistan

\begin{abstract}
Correspondence: Mehwesh Taj, MBBS (Dow), FCPS (Medicine), Fellowship in Clinical Haematology\& Bone Marrow Transplantation,Assistant Professor \& Consultant Clinical Haematologist, CPSP approved Supervisor Clinical Haematology, National Institute of Blood Diseases and Bone Marrow Transplantation, ST 2/A block 17 Gulshan-e-lqbal, Sir Shah Suleman Road, KDA Scheme 24 Karachi, Pakistan, UAN I | |- | 20- | 40, Fax +92-2 I-3482 I 504, Tel +92 300 258|49|, +922I-3482I502-3, Email mehweshfaisal@gmail.com
\end{abstract}

Received: August 02, 2017 | Published: November 24, 2017
Abbreviations: CLL, chronic lymphocytic leukemia; LDT, lymphocyte doubling time; ß2-MG, ß2-microglobulin; ATM, ataxia telangiectasia mutated; TLC, total leucocyte count; SEER, surveillance, epidemiology and end results

\section{Introduction}

Chronic lymphocytic leukemia (CLL) is the frequent type of leukemia in the elderly individuals with an incidence of 4.1 in 100,000 according to the western data. ${ }^{1,2}$ However, about a third of patients are below 60 years of age at diagnosis, and accounts for $30 \%$ of adult leukemia. ${ }^{3}$ It is characterized by clonal proliferation and accumulation of mature B-cells in bone marrow, lymph nodes and lymphoid organs. ${ }^{4}$ The clinical course of patients with chronic lymphocytic leukemia is heterogeneous in nature, with some patients experiencing an aggressive disease progression while others having an indolent course of disease that does not require any treatment except for monitoring of the patient. ${ }^{5}$ Patient's gender and performance status according to ECOG may act as adjuncts to predict the course of disease on individual basis, however, usually these parameters are not useful enough to allow an accurate prognostic counselling for the patient. $^{6,7}$
The Binet and Rai clinical staging systems provide a prediction about the prognosis based on anemia, lymphocytosis, thrombocytopenia and extension of the leukemic infiltration in CLL. ${ }^{8}$ Apart from the prognosis, this particular staging system also lends a hand in the decision of either proceeding with the treatment or just keeping the patient under observation. However these systems do not provide help in predicting that the which patient are lying in the better prognosis group i.e. Stage A of Binet or Grade 0 or 1 of Rai may or may not enter into the bad prognosis group i.e. Stage B Or C of Binet or Grades 2 to 4 of Rai and develop progressive disease in the future. ${ }^{9}$

Below is given the standard clinical staging systems along with the median survival period (Table 1). ${ }^{10}$ Although these staging systems play an important role in deciding the best form of treatment for the patients; other parameters to have been studied in terms of prognostic markers. Few of them include patient characteristics for example gender, age and performance status as well as laboratory parameters showing the tumor burden and disease activity reflected by elevated lymphocyte count, pattern of bone marrow infiltration, lymphocyte doubling time (LDT) and high LDH levels. ${ }^{4}$ A study was done to find the prognostic value of serum markers such as $\beta 2$-microglobulin ( $\beta 2$ MG), soluble CD23 or thymidine kinase in CLL. ${ }^{11}$ Genetic markers of 
tumor cells such as genomic aberrations, genetic abnormalities such as p53 and Ataxia Telangiectasia Mutated (ATM) was also studied for prognostic importance. ${ }^{12}$ The mutation status of the variable segments of immunoglobulin heavy chain genes $\left(\mathrm{V}_{\mathrm{H}}\right)$, or surrogate markers for these factors (CD38, ZAP-70, LPL, etc) ${ }^{13,14}$ was also studied.

Table I Staging with risk stratification and their approximate years of survival

\begin{tabular}{llll}
\hline $\begin{array}{l}\text { Binet staging } \\
\text { system }\end{array}$ & $\begin{array}{l}\text { Rai staging } \\
\text { system }\end{array}$ & $\begin{array}{l}\text { Stratification } \\
\text { of Risk }\end{array}$ & $\begin{array}{l}\text { Median survival } \\
\text { period (years) }\end{array}$ \\
\hline A & 0 & Low risk & $\sim 15$ \\
B & I or II & Intermediate risk & 05-Jul \\
C & III or IV & High risk & 03-Apr \\
\hline
\end{tabular}

As already mentioned above, concurrent genomic and molecular aberrations could change the clinical course, outcome and thus could also influence the treatment plan about every individual patient. Some of the most commonly occurring mutations in B-CLL are being mentioned. Deletions on the chromosome 13, involving band 13q14(del (13q14)) is the single most frequently observed cytogenetic aberration in CLL, occurring in approximately $55 \%$ of all cases and has a benign course ${ }^{15}$ Second most common genetic mutation is the deletion of the long arm of chromosome 11(del(11q)) which can be found in $25 \%$ patients at the time of diagnosis and having advance stage disease and $10 \%$ with early stage disease. This deletion frequently involves band $11 \mathrm{q} 23$ that contains the (ATM) gene and is characterized by bulky lymphadenopathy, rapid progression, and reduced overall survival. ${ }^{16-18}$ Deletions of the short arm of chromosome $17(\operatorname{del}(17 p))$ is the third most common mutation and is found in are found in $5-8 \%$ of patients at the time of diagnosis. This deletion almost always include band $17 \mathrm{p} 13$, where the prominent tumor suppressor gene TP53 is located. CLL patients carrying a del $(17 \mathrm{p})$ show marked resistance against chemotherapy. Mutations of TP53 are found in 4-37\% of patients with CLL, and have been associated with very poor prognosis. ${ }^{19}$

Zeta-associated protein 70 (ZAP-70) is a tyrosine kinase protein normally expressed in $\mathrm{T}$ cells and natural killer cells ${ }^{14}$ and plays an important role in their development, differentiation as well as signalling. B lymphocytes do not express ZAP-70 normally, but it was detected on B-CLL cells. ZAP-70 has been reported to exhibit

Table 2 Binet Staging

\begin{tabular}{llll}
\hline Risk Stratification & Stage & Anemia & Thrombocytopenia \\
\hline Low & A & No & No \\
Intermediate & B & No & No \\
High & C & Yes & Yes \\
\hline
\end{tabular}

\section{Statistical analysis}

Analysis of the data was done on SPSS version 17.0.

\section{Results}

A total of 49patients were included in the study out of which $35(71 \%)$ males and 14(\%) were females. Median age at the time of diagnosis was 58years in our study was (Range: 39-80yrs). Following table gives the demographic characteristics of the patients (Table 3):

At the time of diagnosis, according to Binet staging 04(8.1\%) patients were categorized in Stage A, 04(8.1\%) patients in stage B and $17(34.6 \%)$ patients in Stage $\mathrm{C}$ were noted. On bone marrow preference in CLL unmutated Ig $\mathrm{V}_{\mathrm{H}}$-type B-cell clones. The ligation of B-cell receptor on CLL cells that express ZAP-70 is associated with increased tyrosine kinase activity leading to excessive phosphorylation. This finding has led to the implication that the presence of ZAP-70 in CLL B-cell clones reflects an "activation" state of the clone and may be related to the poorer outcome in those patients. ${ }^{20,21}$

Therefore, there is a need to identify markers that may help towards a much more accurate prediction of prognosis for CLL patients. Also a risk versus benefit evaluation based on an individual's disease characteristics would be desirable so that the grading and eventually the treatment is tailored according to them. In this paper we review the available tools for patient risk stratification and discuss how these tools can be used in routine clinical practice to individualize patient's prognosis and thus the treatment.

\section{Material and methods}

49patients diagnosed with Chronic Lymphocytic Leukaemia at our centre over a period of three and a half (3.5) years from January 2010 to July 2013 were selected for this study. The diagnosis of CLL was established by blood counts, differential counts, a blood smear, and immunophenotyping. The blood counts showed high Total Leucocyte Count (TLC) with differential exhibiting lymphocytosis (more than $5 \times 10^{9} / \mathrm{L}$ in peripheral smear and more than $30 \%$ in the bone marrow. Patients haemoglobin and platelets level were also recorded and haemoglobin less than $10 \mathrm{gm} / \mathrm{dl}$ was labelled as anemia and platelets less than $100 \times 10^{9} / \mathrm{L}$ was considered as thrombocytopenia. Immunophenotypically the leukemic cells were checked for the presence of CD45, CD34, Tdt, CD4, CD5, CD7, CD 10, CD20, CD23, CD79a, CyclinD1.

ZAP-70 was also checked for immunophenotypically. The leukemic cells showed positivity for CD45, CD5, CD20, CD23, CD79a and CyclinD1 to reach to a diagnosis of CLL. Clinicopathological parameters were retrieved retrospectively from patient's records. Presence of any visceromegaly or/and lymphadenopathy was also noted. Furthermore presence of patient's constitutional symptoms such as fever, weight loss and sweating were recorded. For staging of the disease at the time of diagnosis, only the Binet clinical staging was used in our study (Table 2).

\section{Lymphoid regions (Lymphadenopathy/Spleno- or Hepatomegaly) \\ $<2$ Lymphoid regions involved \\ $>2$ Lymphoid regions involved \\ $>2$ Lymphoid regions involved}

biopsy, pattern of infiltration placed $07(14.2 \%)$ patients with Nodular infiltration, $14(28.5 \%)$ patients with Interstitial, 07(14.2\%) patients with Interstitial to diffuse and 21(42.8\%) patients with diffuse pattern of infiltration. We also grouped the patients according to their staging as well as their pattern of infiltration (Table 4). The table below gives our results.

Immunohistochemistry panel showed CD45, CD5, CD20, CD23 and CD79a positivity in almost all the cases. ZAP 70positivity was seen in $17(34.7 \%)$ patients. Presence of Zap-70 was also seen according to the respective stages (Table 5). In our study out of 49 patients 10 were tested for del $17 \mathrm{p}$ and their frequency seen according to the staging at the time of diagnosis is given (Table 6) 
Table 3 Demographic characteristics

\begin{tabular}{ll}
\hline Demographic Characteristics & Frequency (\%) \\
\hline Median age (Males) & 60 years \\
Median age (Females) & 54 years \\
Lymphadenopathy & $16(32.6)$ \\
Splenomegaly & $12(24.4)$ \\
Hepatomegaly & $07(14.2)$ \\
Fever & $19(38.7)$ \\
Weight loss & $15(30.6)$ \\
Sweating & $02(04)$ \\
\hline
\end{tabular}

Table 4 Pattern of Infiltration and their staging

\begin{tabular}{|c|c|}
\hline Staging & Frequency (\%) \\
\hline \multicolumn{2}{|c|}{ Nodular $(\mathbf{N}=7)$} \\
\hline Stage A & $05(10.2)$ \\
\hline Stage B & $01(2.0)$ \\
\hline Stage C & $01(2.0)$ \\
\hline \multicolumn{2}{|c|}{ Interstitial $(\mathrm{N}=14)$} \\
\hline Stage A & $10(20.4)$ \\
\hline Stage B & $02(4.1)$ \\
\hline Stage C & $02(4.1)$ \\
\hline \multicolumn{2}{|c|}{ Interstitial to Diffuse $(\mathrm{N}=07)$} \\
\hline Stage A & $0 \mathrm{OI}(2.0)$ \\
\hline Stage B & 0 \\
\hline Stage C & $06(12.2)$ \\
\hline \multicolumn{2}{|c|}{ Diffuse (N=2I) } \\
\hline Stage A & $04(8.2)$ \\
\hline Stage B & $01(2.0)$ \\
\hline Stage C & 16(32.7) \\
\hline
\end{tabular}

Table 5 ZAP-70 and the respective staging

\begin{tabular}{ll}
\hline Stage & Frequency (\%) \\
\hline Stage A & $07(41.2)$ \\
Stage B & $01(5.9)$ \\
Stage C & $09(52.9)$ \\
\hline
\end{tabular}

Table 6 Del I7p and their frequency

\begin{tabular}{ll}
\hline Stage & Frequency (\%) \\
\hline Stage A & $01(10 \%)$ \\
Stage B & 0 \\
Stage C & $02(20 \%)$ \\
\hline
\end{tabular}

\section{Discussion}

Rai et al. ${ }^{22}$ and Binet et al. ${ }^{23}$ are the two clinical staging systems that have been established as standards to estimate the prognosis of CLL in patients. The disease, however, in previous study shows a heterogeneous pattern in individual patients with similar staging. ${ }^{9}$ Several studies have been done to identify various other clinical and biological factors that may have prognostic importance in addition to the classic assessment. The Binet and Rai clinical staging systems base their prediction of prognosis in B-CLL on anemia, thrombocytopenia and extension of the leukemic infiltration. ${ }^{4}$ The other parameters to have been studied in terms of prognostic markers are patient performance status according to ECOG along with the laboratory parameters that show the tumor burden and disease activity reflected by elevated lymphocyte count, pattern of bone marrow infiltration, lymphocyte doubling time (LDT) and high LDH levels. ß2-microglobulin (B2-MG) and soluble CD23 have also been studies as prognostic markers in CLL. Recently, emphasis have been placed on the presence of genetic markers of tumor cells such as p53 and ATM and their implications if present as they give a poorer outcome to the patients disease. The variable segments of immunoglobulin heavy chain genes $\left(\mathrm{V}_{\mathrm{H}}\right)$ and their markers such as $\mathrm{CD} 38$, ZAP-70 was also studied. In this study we evaluate the correlation between some of the above mentioned prognostic factors in CLL patients with their Binet staging and thus the prognosis of these patients.

In United States, the Surveillance, Epidemiology and End Results (SEER) in 2013 showed 67years and 72years as median age at the time of diagnosis in men and women respectively. In our study, the median age at diagnosis is 60years for men and 54years for women. ${ }^{24-26}$ The western data showed a median age of approximately 70years at the time of diagnosis for entire group ${ }^{27}$ while in our study 58years was the median age for the entire group. Our study showed a male preponderance (M:F ratio, 3.3:1) in a study group of 49patients which was similar to the western data also showing a male preponderance (M:F ratio, 1.7:1) although the difference between the proportions of the two genders in the western data was considerably less. Our study however was shown to negate the results conducted in Niger which comprised of sixty patients and showed female preponderance (M:F ratio, 1:3) ${ }^{28}$ Although CLL is a disease of the elderly and its incidence as well as the prevalence is directly proportional to increasing age, recent studies show a rise in the proportion of younger patients due to frequent blood testing. ${ }^{29}$ This same study had $15 \%$ patients who were below the age of 40years which our study also showed as we had $3(6 \%)$ patients below the age of 40years. ${ }^{28}$

An extensive study of 329patients led to bone marrow histology being designated as the beneficial predictor of prognosis with the diffuse pattern of infiltration being an indicator of poor prognosis. ${ }^{30}$ Our study also showed that the majority of the patients who were found out to have diffuse pattern of infiltration also were placed in the stage $\mathrm{C}$ at the time of diagnosis confirming the results of the related study that diffuse infiltration leads to poorer outcome and minimum survival. Studies also show that the presence of ZAP70 leads to excessive tyrosine kinase activity and thus to increased phosphorylation. This increased activity keeps the leukemic cells in an "activated" state, giving the disease an aggressive pattern to follow as well as poorer outcome in patients..$^{20,21}$ Our study also showed that out of the 17 patients who were found to be ZAP-70 positive, 09 patients were placed in Stage $C$ at the time of diagnosis leading to the conclusion that the presence of ZAP-70 gives patients a poorer survival rate. Further prospective studies are required to evaluate the importance of the genetic aberrations as markers of prognosis in CLL. 


\section{Conclusion}

In our center most of the patients were in Binet stage $\mathrm{C}$. About onethird of the total patients showed ZAP-70 positivity which indicates early progression to advance stage and need of immediate therapeutic intervention. All the CLL patients should not only be staged according to Rai/Binet Staging Systems; but also other genomic markers like Cytogenetic studies should be carried out to stratify the management accordingly.

\section{Financial disclosure}

No financial disclosure to be documented

\section{Acknowledgements}

None.

\section{Conflict of Interest}

The authors have declared that no conflict of interest existed.

\section{References}

1. Jemal A, Siegel R, Ward E, et al. Cancer statistics. CA Cancer J Clin. 2007;57(1):43-66.

2. Surveillance Epidemiology and End Results Cancer Statistics review. Spain: National Cancer Institute; 2010.

3. Shanafelt TD. Predicting clinical outcome in CLL: how and why. Hematology Am Soc Hematol Educ Program. 2009;1:421-429.

4. Rozman C, Montserrat E. Chronic lymphocytic leukemia. $N$ Engl J Med. 1995;333(16):1052-1057.

5. Mozaheb Z, Hasanzadeh NazarAbadi MH, et al. Chronic lymphocytic leukemia and prognostic factor. Asian Pac $J$ Cancer Prev. 2012;13(7):3009-3013.

6. Neil E Kay, Tait D Shanafelt. Shanafelt, MD. Prognostic factors in chronic lymphocytic leukemia. Current Hematologic Malignancy Reports. 2007;2(1):49-55.

7. Oken MM, Creech RH, Tormey DC, et al. Toxicity and response criteria of the eastern cooperative oncology group. Am J Clin Oncol. 1982;5(6):649-655.

8. Saka B, Aktan M, Sami U, et al. Prognostic importance of soluble CD23 in B-cell chronic lymphocytic leukemia. Clin Lab Haematol. 2006;28(1):30-35.

9. Kröber A, Seiler T, Benner A, et al. $\mathrm{V}_{(\mathrm{H})}$ mutation status, CD38 expression level, genomic aberrations, and survival in chronic lymphocytic leukemia. Blood. 2002;100(4):1410-1416.

10. Montserrat E. New Prognostic Markers in CLL. Hematology Am Soc Hematol Educ Program. 2006;2006:279-284.

11. Hallek M, Langenmayer I, Nerl C, et al. Elevated serum thymidine kinase levels identify a subgroup at high risk of disease progression in early, non-smoldering chronic lymphocytic leukemia. Blood. 1999;93(5):1732-1737.

12. Montserrat E. Classical and new prognostic factors in chronic lymphocytic leukemia: where to now? Hematol J. 2002;3(1):7-9.

13. Oscier DG, Gardiner AC, Mould SJ, et al. Multivariate analysis of prognostic factors in CLL: clinical stage, IGVH gene mutational status, and loss or mutation of the p53 gene are independent prognostic factors. Blood. 2002;100(4):1177-1184.
14. Crespo M, Bosch F, Villamor N, et al. ZAP-70 expression as a surrogate for immunoglobulin-variable-region mutations in chronic lymphocytic leukemia. $N$ Engl J Med. 2003;348(18):1764-1775.

15. Calin GA, Dumitru CD, Shimizu M, et al. Frequent deletions and downregulation of micro- RNA genes miR15 and miR16 at 13 q14 in chronic lymphocytic leukemia. Proc Natl Acad Sci USA. 2002;99(24):15524-15529.

16. Zenz T, Mertens D, Küppers R, et al. From pathogenesis to treatment of chronic lymphocytic leukaemia. Nat Rev Cancer. 2010;10(1):37-50.

17. Quesada V, Conde L, Villamor N, et al. Exome sequencing identifies recurrent mutations of the splicing factor SF3B1 gene in chronic lymphocytic leukemia. Nat Genet. 2011;44(1):47-52.

18. Döhner H, Stilgenbauer S, James MR, et al. 11q deletions identify a new subset of B-cell chronic lymphocytic leukemia characterized by extensive nodal involvement and inferior prognosis. Blood. 1997;89(7):2516-2522.

19. Zenz T, Vollmer D, Trbusek M, et al. TP53 mutation profile in chronic lymphocytic leukemia: Evidence for a disease specific profile from a comprehensive analysis of 268 mutations. Leukemia. 2010;24(12):20722079.

20. Crespo M, Villamor N, Giné E, et al. ZAP-70 expression in normal pro/ pre B cells, mature B cells, and in B-cell acute lymphoblastic leukemia. Clin Cancer Res. 2006;12(3 Pt 1):726-734.

21. Nolz JC, Tschumper RC, Pittner BT, et al. ZAP-70 is expressed by a subset of normal human B-lymphocytes displaying an activated phenotype. Leukemia. 2005;19(6):1018-1024.

22. Rai KR, Sawitsky A, Cronkite EP, et al. Clinical staging of chronic lymphocytic leukemia. Blood. 1975;46(2):219-234.

23. Binet JL, Auquier A, Dighiero G, et al. A new prognostic classification of chronic lymphocytic leukemia derived from a multivariate survival analysis. Cancer. 1981;48(1):198-206.

24. Molica S. Sex differences in incidence and outcome of chronic lymphocytic leukemia patients. Leuk Lymphoma. 2006;47(8):14771480.

25. Morton LM, Wang SS, Devesa SS, et al. Lymphoma incidence patterns by WHO subtype in the United States, 1992-2001. Blood. 2006;107(1):265276.

26. Watson L, Wyld P, Catovsky D. Disease burden of chronic lymphocytic leukemia within the European Union. Eur J Haematol. 2008;81(4):253258 .

27. Moreno C, Montserrat E. Genetic lesions in chronic lymphocytic leukemia: what's ready for prime time use? Haematologica. 2010;95(1):12-15.

28. Omoti CE, Awodu OA, Bazuaye GN. Chronic lymphoid leukaemia: clinico-haematological correlation and outcome in a single institution in Niger Delta region of Nigeria. Int J Lab Hematol. 2007;29(6):426-432.

29. Mauro FR, Foa R, Giannarelli D, et al. Clinical characteristics and outcome of young chronic lymphocytic leukemia patients: A single institution study of 204 cases. Blood. 1999;94(2):448-454.

30. Rozman C, Montserrat E, Rodríguez-Fernández JM, et al. Bone marrow histologic pattern-the best single prognostic parameter in chronic lymphocytic leukemia: a multivariate survival analysis of 329 cases. Blood. 1984;64(3):642-648. 